\title{
Two commentaries on review of characteristics of child dental neglect
}

\author{
Abstracted from \\ Bhatia SK, Maguire SA, Chadwick BL, Hunter ML, Harris JC, Tempest V, Mann MK, Kemp AM. \\ Characteristics of child dental neglect: A systematic review. J Dent 2014; 42: 229-239. \\ Address for correspondence: Dr S K Bhatia, University Dental Hospital, School of Dentistry, Cardiff University, Cardiff, CF14 4EA \\ E-mail: bhatiask@cardiff.ac.uk
}

\section{Question: What are the characteristics of child dental neglect?}

Data sources ASSIA, EBSCO - CINAHL, Cochrane CENTRAL, EMBASE, ERIC, MEDLINE, SCOPUS, OpenGrey, Proquest - Social Services/ Sociological Abstracts, Web of Knowledge - Conference Proceedings Citation Index and ISI Science Citation/ Social Science Citation Index. There were no language restrictions and authors were contacted for primary data and additional information when required.

Study selection Studies were considered eligible if they were primary studies of children ( 0 to 18 years) with confirmed oral neglect and with detail of dental, intra- and extra-oral features. Exclusion criteria included; studies of sexual or physical abuse or those where there was oral neglect suspected but no detail was given. All characteristics associated with dental neglect were considered outcomes.

Data extraction and synthesis Each article was independently reviewed by two reviewers from a panel of 22 trained reviewers with

\section{Some clarification of trigger signs for dental neglect}

Neglect is the most prevalent form of child abuse yet it is in itself a neglected topic, being superseded in the press by physical and sexual assault. ${ }^{1}$ Dental neglect can be defined as: 'the failure of a parent or guardian to meet the child's basic oral health needs such that the child enjoys adequate function and freedom from pain and infection, where reasonable resources are available to family or caregiver. $^{2}$

Health care professionals outside of the dental team, it seems, are reluctant to look inside children's mouths. ${ }^{1}$ Despite attempts to promote early identification of neglect through the Department of Health's 'Child Protection and the Dental Team' booklet, and the 'Getting It Right For Every Child' policy for Scotland, dentists have been reluctant to report suspected cases of neglect and abuse.

Likely reasons include lack of training or guidance and fear of impact on their practice, risk of litigation or family violence towards themselves or the child. ${ }^{3}$ Yet, dental professionals should be able to identify dental neglect, not only as a problem in itself, but also as it may be the first sign of general childhood neglect. ${ }^{4}$ a third reviewer resolving any disagreement. A specifically designed tool for quality assessment was used.

Results Three thousand eight hundred and sixty-three citations and abstracts were scanned, 83 studies were reviewed and nine (with 1595 children aged 0 to 15 years) met the inclusion criteria, including two case control studies. There were not enough data to allow metaanalysis to be carried out.

The features most commonly recorded as being characteristics of dental neglect were; failure to seek, or delay in seeking dental treatment, failure to follow advice or complete treatment, and this often co-existed with an adverse impact of dental neglect on the child including pain and swelling. A 'threshold' level of dental caries, that allowed dental neglect to be defined, could not be established from the available literature.

Conclusions There was limited literature on this topic. The main characteristics of dental neglect highlighted were failure/delay in seeking dental care, with adverse consequences. However, due to a lack of research and additionally, a deficiency in the data on precise clinical features, it was not possible to make the distinction between dental caries and dental neglect.

This study was carried out by Core Info Cardiff Child Protection Systematic Reviews: an experienced team both in this clinical field and with systematic reviews.

Using a comprehensive literature search with strict inclusion criteria they identified nine articles outlining the common characteristics of dental neglect. These studies consistently reported that signs of dental neglect could include: failure to seek or a delay in seeking dental treatment; failure to follow dental advice; failure to administer medicine and provide basic oral care. Of these nine studies, none was set in the UK.

Six of the studies were North American and the remaining three from Italy, Sweden and Australia. Although all are Western cultures, there may be subtle differences in what is considered neglect between countries.

The review also addresses the difficult question; when does dental caries become dental neglect? Unfortunately, no evidence was found to support a definitive threshold number of lesions which, when reached, becomes a diagnosis of neglect. For example, one of 
the case studies included suggests that a diagnosis of early childhood caries should alert the clinician to the possibility of neglect. Yet this may only be an alert to a lack of knowledge or skills in the parent/caregiver rather than a neglectful attitude.

Although this systematic review clarifies the key signs to trigger consideration of dental neglect: failure to seek or a delay in seeking dental treatment; failure to follow dental advice; failure to administer medicine and provide basic oral care, more importantly it highlights what still needs to be done.

Development of a screening tool combining caries rate at that age, with some of the listed dental neglect characteristics, may be helpful. This could trigger an alert for higher risk families to be referred for multi-disciplinary team input of this complex issue.

- Child dental neglect is an important topic for the dental team as it may be the first signs of general neglect.

- Identifying neglect can be difficult; the clinician should famil- iarise themselves with the characteristics and refer if they have concerns.

Gregory Soustera and Nicola Innes ${ }^{b}$

${ }^{a}$ Dundee Dental Hospital, Park Place, Dundee, Scotland, UK ${ }^{b}$ University of Dundee, Unit of Dental and Oral Health, Park Place, Dundee, Scotland, UK

1. Bradbury-Jones C, Innes N, Evans D, Ballantyne F, Taylor J. Dental neglect as a marker of broader neglect: a qualitative investigation of public health nurses' assessments of oral health in preschool children. BMC Public Health 2013; 13 370

2. Bhatia SK, Maguire SA, Chadwick BL, et al. Characteristics of child dental neglect: A systematic review. J Dent 2014; 42: 229-239.

3. Cairns AM, Mok JY, Welbury RR. The dental practitioner and child protection in Scotland. Br Dent / 2005; 199: 517-520.

4. Harris JC, Elcock C, Sidebotham PD, Welbury RR. Safeguarding children in dentistry: 2. Do paediatric dentists neglect child dental neglect? Br Dent J 2009, 206: $465-470$

Evidence-Based Dentistry (2014) 15, 2-4. doi: 10.1038/sj.ebd.6400996

\section{Systematic review highlights difficulty in clearly identifying dental neglect in children}

The United Nations Convention on the Rights of the Child states that children are entitled to a standard of living that supports their physical, mental, spiritual, moral and social development. Parent(s) or others responsible for the child should, within their abilities and financial capacities, pursue the living conditions necessary for the child's development. ${ }^{1}$

Unfortunately, child maltreatment, including abuse and neglect, remains a problem in modern society and this neglect has dental and oral health manifestations.

The General Dental Council (GDC) published Standards for the Dental Team ${ }^{2}$ in September 2013, which set out the responsibilities of dental team members to ensure they take action where there is concern over possible abuse of children and vulnerable adults. The standards go on to state:

- You must raise any concerns you may have about the possible abuse or neglect of children or vulnerable adults. You must know whom to contact for further advice and how to refer concerns to an appropriate authority such as your local social services department.

- You must find out about local procedures for the protection of children and vulnerable adults. You must follow these procedures if you suspect that a child or vulnerable adult might be at risk because of abuse or neglect.

Moreover, there are a number of sources of child protection guidance for health professionals, including: the Department of Health;3 Scottish Government ${ }^{4}$ and the Scottish Clinical Effectiveness Programme (SDCEP) guidance for the dental team on caries prevention and management in children ${ }^{5}-$ which is currently being updated taking into account the new legislative background of the Children and Young People (Scotland) Bill. ${ }^{6}$

The subject of dental neglect remains poorly and inconsistently understood. The aim of the paper was to determine the scientific evidence underpinning the characteristics of dental neglect in children.
A clear, focused question was set 'What are the features of oral neglect in children?' and for the purposes of the literature search dental neglect was defined as: 'Neglect refers to the failure of a parent or guardian to meet a child's basic oral health needs, such that the child enjoys adequate function and freedom from pain and infection, where reasonable resources are available to the family or caregiver'.

A robust global systematic literature search was conducted (including 15 databases from 1960-2012 and including unpublished and grey literature).

Studies were assigned a grade on the basis of their quality standards, although it is not clear within the text how this was assessed or the significance of the quality grades. Nine studies including data on a reasonably high number of children $(n=1595)$ were eventually included in the narrative synthesis analysis. There was high heterogeneity of the included studies with varying definitions of dental neglect and oral criteria adopted.

The results suggested that there were common features of dental neglect including: (i) failure to seek or delay in seeking dental treatment; (ii) failure to comply with and or complete treatment; (iii) failure to implement basic oral care (oral hygiene, diet and dental attendance); (iv) symptoms such as pain and swelling. The issue of differentiating dental caries from dental neglect was highlighted and there were no specific patterns to dental caries associated with neglect.

The review is a good start at systematically appraising the limited literature in this area. However, the main disappointment in the review (or rather the evidence available) was that it was unable to set out a hierarchy of concern indicators, which could guide dental practitioners in how and when these concerns should be raised or shared more widely.

With legislative changes underscoring the importance and priority of this area, the forthcoming SDCEP guidance update will further help practitioners navigate these difficult decisions, but it will have to rely on best practice rather than best evidence. 


\section{PAEDIATRIC DENTISTRY}

Fiona Noble ${ }^{a}$, Jamie Dickie ${ }^{\mathrm{a}}$ and David I Conway ${ }^{\mathrm{b}}$ ${ }^{a}$ Glasgow Dental Hospital and School, Glasgow, Scotland, UK ${ }^{b}$ University of Glasgow Dental School, Glasgow, Scotland, UK

1. UNICEF. The UN Convention on the Rights of the Child. https://www.unicef.org.uk/ UNICEFs-Work/Our-mission/UN-Convention/

2. Standards for the Dental Team. General Dental Council (2013) https://www.gdcuk.org/Dentalprofessionals/Standards/Documents/Standards\%20for\%20the\%20 Dental\%20Team.pdf

3. Child Protection and the Dental Team. Department of Health (2013) http://www. cpdt.org.uk

4. Child Protection Guidance for Health Professionals. Scottish Government (2013) http://www.scotland.gov.uk/Resource/0041/00411543.pdf

5. Scottish Dental Clinical Effectiveness Programme (2010) Prevention and Management of Dental Caries in Children http://www.sdcep.org.uk/index. asp $\mathrm{x}$ o $=2332$

6. Children and Young People (Scotland) Bill (2014) http://www.scotland.gov.uk/
Topics/People/Young-People/legislation

3. Cahlin B], Dahlström L. No effect of glucosamine sulfate on osteoarthritis in the temporomandibular joints - a randomized, controlled, short-term study. Oral Surg Oral Med Oral Pathol Oral Radiol Endod 2011; 112: 760-766.

4. Manfredini D, Rancitelli D, Ferronato G, Guarda-Nardini L. Arthrocentesis with or without additional drugs in temporomandibular joint inflammatorydegenerative disease: comparison of six treatment protocols. / Oral Rehabil 2012 39: $245-251$

5. Guarda-Nardini L, Cadorin C, Frizziero A, Ferronato G, Manfredini D. Comparison of 2 hyaluronic acid drugs for the treatment of temporomandibular joint osteoarthritis. J Oral Maxillofac Surg 2012; 70: 2522-2530.

6. Palla S, Farella M. External validity: a forgotten issue? / Orofac Pain. 2009; 23 297-298.

7. Manfredini D, Guarda-Nardini L, Winocur E, Piccotti F, Ahlberg J, Lobbezoo F. Research diagnostic criteria for temporomandibular disorders: a systematic review of axis I epidemiologic findings. Oral Surg Oral Med Oral Pathol Oral Radiol Endod 2011; 112: 453-462.

Evidence-Based Dentistry (2014) 15, 2-4. doi: 10.1038/sj.ebd.6400978 